\title{
Prevalencia de Síndrome Metabólico EN NIÑOS Y ADOLESCENTES DE AMÉRICA
}

\author{
Romain Pierlot', Estela Cuevas-Romero², Jorge Rodríguez-Antolín², \\ Pablo Méndez-Hernández ${ }^{3}$ y Margarita Martínez-Gómez ${ }^{2,4 *}$ \\ ${ }^{1}$ Doctorado en Ciencias Biológicas, Universidad Autónoma de Tlaxcala. ${ }^{2}$ Centro Tlaxcala de Biología de \\ la Conducta, Universidad Autónoma de Tlaxcala. ${ }^{3}$ Dirección de Enseñanza e Investigación, Secretaría \\ de Salud de Tlaxcala. ${ }^{4}$ Departamento de Biología Celular y Fisiología. Instituto de Investigaciones \\ Biomédicas, Universidad Nacional Autónoma de México. Unidad Periférica Tlaxcala. Carretera Tlaxcala- \\ Puebla Km. 1.5, C.P. 90070, Tlaxcala, México. E-mail: *marmag@biomedicas.unam.mx
}

\begin{abstract}
RESUMEN
Objetivo: Analizar la prevalencia del Síndrome Metabólico (SM) y sus componentes en niños y adolescentes del continente Americano.

Método: La recopilación de la información se tomó de artículos científicos de los años 2008 al 2016 que aparecen en las bases de datos PubMed, Europe PMC y SciELO. Se incluyeron estudios que muestran datos cuantitativos de los componentes del SM en niños y/o adolescentes entre 4 y 19 años de edad.

Resultados: Se analizaron 23 estudios realizados en 12 países del continente americano, de los cuales sólo 3 consideraron el factor edad. Los componentes del SM más prevalentes fueron la obesidad y las dislipidemias. Mientras que los menos prevalentes fueron hiperglicemia e hipertensión. La hipoalipoproteinemia, la hipertensión y el SM fueron más frecuentes en hombres que en mujeres. Pocos estudios analizaron factores de riesgo para el SM. Conclusión: La presencia del SM y sus componentes en niños y adolescentes americanos es variable. La variabilidad es también observada en países de otros continentes. Esta revisión evidencia la necesidad de definir los criterios de diagnóstico del SM en niños y jóvenes, y la urgencia de diseñar estrategias de prevención de las alteraciones metabólicas en edades tempranas, involucrando la participación de la familia, la sociedad y las instituciones públicas.
\end{abstract}

Palabras Clave: diabetes, dislipidemias, hipertensión arterial, obesidad, síndrome metabólico.

\section{Prevalence of Metabolic Syndrome in American children and adolescents}

\begin{abstract}
Objective: To analyze the prevalence of Metabolic Syndrome (MS) and its components in children and adolescents in American continent.

Methods: Information was collected from scientific papers published from 2008 to 2016 in PubMed, Europe PMC, and SciELO databases. Only those studies showing quantitative data from MS components in 4-19 years old children and adolescents were included.

Results: Twenty-three studies in 12 countries in American continent were analyzed, only three studies considered age factor. Obesity and dyslipidemias were highly prevalent; while hyperglycemia and hypertension had a low prevalence. Hypoalipoproteinemia, hypertension and MS were more frequent in males than in females. Few studies analyzed risk factors for MS.

Conclusion: Similarly, to other continents, the presence of MS and its components in American children and adolescents is variable. This review shows the necessity to have clear diagnostic criteria for MS in children and adolescents, as well as the urgency to get preventive strategies for metabolic alterations at young age, involving family, society, and public institutions.
\end{abstract}

Key Words: diabetes, dyslipidemias, hypertension, obesity, metabolic syndrome.

Nota: Artículo recibido el 09 de abril de 2016 y aceptado el 25 de octubre de 2016. 


\section{INTRODUCCIÓN}

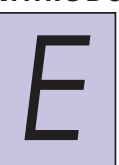

1 Síndrome Metabólico (SM) es una combinación de presión arterial alta, obesidad abdominal, dislipidemias, intolerancia a la glucosa y/o resistencia a la insulina, así como un aumento de moléculas pro-inflamatorias ${ }^{1,2}$. Su diagnóstico en niños y adultos suele hacerse con el mismo criterio, reunir al menos 3 de los componentes ya descritos, aunque con ciertas variaciones en los puntos de corte ${ }^{2,3}$. Así como en los adultos, el SM en niños es un factor de riesgo para el desarrollo de diabetes mellitus 2 (DM2), hipertensión, enfermedades cardiovasculares y daño renal ${ }^{1,2}$. Además, la presencia del SM en la pubertad puede condicionar la fertilidad durante la edad adulta y está asociado a la presencia de ciclos menstruales irregulares y ovario poliquístico ${ }^{4,5}$. El impacto del SM en la salud reproductiva de los niños ha sido poco estudiada, sin embargo, en hombres adultos la obesidad se asocia con infertilidad, hipogonadismo, niveles bajos de testosterona, bajo conteo espermático e incluso un deterioro en el desarrollo embrionario que aumenta la tasa de aborto ${ }^{6}$.

La raza, es un factor que influye fuertemente en el desarrollo del SM a edades tempranas. Así, niños y jóvenes hispanos, africanos y asiáticos tienen una mayor prevalencia del SM que los no hispanos ${ }^{2}$. Otro factor importante, es el estado de la salud materna durante el desarrollo intrauterino del feto que puede influir en los cambios epigenéticos y en el desarrollo de alteraciones metabólicas en los hijos ${ }^{7}$. En niños y adolescentes con predisposición genética o epigenética, una buena nutrición y actividad física podrían retardar el desarrollo o evolución de las alteraciones metabólicas ${ }^{1}$. Dado que los niños, y en menor medida los jóvenes, dependen aún de la familia, las alteraciones metabólicas a esta edad se relacionan con los cambios en la alimentación y por actividades de los padres que favorecen el consumo de alimentos procesados con alto contenido en carbohidratos, grasa y sal ${ }^{1}$. Por ello, la obesidad parental se considera un predictor de la obesidad de los hijos ${ }^{8}$.

Los adolescentes son un grupo de gran interés en las políticas de salud pública, debido a que en esta etapa de la vida se desarrollan actitudes y prácticas de comportamiento relacionadas con la salud, que tienen alta posibilidad de permanecer durante toda la vida $^{9}$, determinándose así el estatus de salud que los adolescentes tendrán en la edad adulta ${ }^{10}$. Se ha mostrado que más de la mitad de las muertes relacionadas con las enfermedades crónicas están asociadas a comportamientos, que comienzan durante la adolescencia, y son difíciles de cambiar ${ }^{11}$, como el consumo de tabaco, alcohol, mala calidad de la alimentación y falta de actividad física $^{12}$. Por otro lado, la pubertad y la adolescencia son dos etapas complejas de la vida. Los jóvenes buscan su autonomía y prestan mayor atención a su apariencia por lo que un aumento de peso o un deterioro, son factores para reducir su autoestima, el aislamiento y la fatiga, así como la presencia de episodios depresivos ${ }^{1}$.
Por todo lo anteriormente descrito, consideramos que existe la necesidad de conocer cuál es la tendencia general de las alteraciones metabólicas en los niños y adolescentes de los distintos países del continente Americano, con el objetivo de denotar su magnitud, describir las prevalencias del SM, las causas, factores de riesgo y sus componentes.

\section{Método}

La recopilación de la información se tomó de artículos científicos de los años 2008 al 2016 contenidos en las bases de datos PubMed, Europe PMC y SciELO. Los criterios de integración de los artículos fueron: que éstos se enfocaran en niños y/o adolescentes entre 4 a 19 años de edad, y mostraran datos cuantitativos sobre los componentes del $\mathrm{SM}$, como niveles de obesidad y sobrepeso, hipertensión arterial, hiperglucemia y dislipidemias, e información sobre la prevalencia del SM.

En lapresenterevisión se incluyeron 23 estudios que consideraron los criterios propuestos por la National Cholesterol Education Program Adult Treatment Panel III (NCEP ATP III) ${ }^{13}$ y por la Federación Internacional de la Diabetes (IDF, por sus iniciales en inglés $)^{14}$ presentados en la Tabla I.

\section{Resultados}

Los datos reportados son el resultado del análisis de 23 artículos que cumplieron con los criterios de inclusión, y que corresponden a 12 países de América: Argentina ${ }^{15-18}$, Brasil $^{19}$, Canadáa ${ }^{20-21}$, Chile ${ }^{22-24}$, Colombia ${ }^{25-27}$, Ecuador ${ }^{28}$, Estados Unidos de América (EUA) ${ }^{29}$, Guatemala ${ }^{30}$, México $^{31-34}$, Paraguay ${ }^{35}$, Perú ${ }^{36}$ y Venezuela ${ }^{37}$. Del total de estudios analizados, solamente 21 trabajos mostraron prevalencias generales de obesidad abdominal, 18 de ellos tuvieron prevalencias de hiperglucemia, otros 18 indicaron las prevalencias de hipertrigliceridemia, 19 las prevalencias de hipoalfalipoproteinemia (HDL-C bajo), 20 más midieron prevalencias de hipertensión arterial, y 18 analizaron prevalencias del SM. Solamente un estudio mostró resultados por grupos étnicos y sólo 12 diferenciaron las prevalencias por género. Todos los estudios reportaron datos de muestras urbanas, excepto el de Ochoa-Avilés que se refiere a los jóvenes de áreas rurales.

El análisis de las prevalencias del SM y sus componentes en niños y adolescentes del continente americano fueron elevadas, casi una cuarta parte presentaron niveles bajos de HDL-C, aproximadamente una quinta parte con niveles de triglicéridos elevados y obesidad abdominal, además uno de cada 10 niños y adolescentes presentaron tensión arterial elevada, alrededor del $7 \%$ con hiperglucemia y SM. De manera general, los componentes más prevalentes fueron la obesidad y las dislipidemias (hipertrigliceridemia y HDL-C); mientras que, la hiperglicemia y la hipertensión mostraron una menor prevalencia (Figuras 1a-f). 


\begin{tabular}{|c|c|c|c|c|c|c|}
\hline Criterio & Obesidad & Triglicéridos & HDL-C & $\begin{array}{c}\text { Hipertensión } \\
\text { Arterial }\end{array}$ & Glucosa & SM \\
\hline $\begin{array}{c}\text { National } \\
\text { Cholesterol } \\
\text { Education Program } \\
\text { Adult Treatment } \\
\text { Panel III } \\
\text { (NCEP ATP III) }\end{array}$ & $\begin{array}{c}\text { Percentil }>90 \text { de } \\
\text { circunferencia de } \\
\quad \text { cintura }\end{array}$ & $\begin{array}{c}\text { Niños: } \\
\text { percentil } \geq 75 \\
\\
\text { Adolescentes: } \\
\geq 110 \mathrm{mg} / \mathrm{dL} \text { o } \\
\text { percentil }>95\end{array}$ & $\begin{array}{l}\text { Niños: } \\
\text { percentil } \\
\quad \leq 25 \\
\text { Adolescentes: } \\
\leq 40 \mathrm{mg} / \mathrm{dL}\end{array}$ & $\begin{array}{l}\text { Presión arterial } \\
\text { sistólica o } \\
\text { diastólica } \\
\text { percentil } \geq 90\end{array}$ & $\begin{array}{c}\text { Glucemia basal } \\
>100 \mathrm{mg} / \mathrm{dL} \mathrm{o} \\
\text { postprandial }> \\
140 \mathrm{mg} / \mathrm{dL}\end{array}$ & $\begin{array}{l}\text { Si el infante o el joven } \\
\text { presenta al menos } 3 \text { de } \\
\text { esos componentes }\end{array}$ \\
\hline $\begin{array}{l}\text { International } \\
\text { Diabetes } \\
\text { Federation } \\
\text { (IDF) }\end{array}$ & $\begin{array}{c}\text { Percentil }>90 \text { de } \\
\text { circunferencia de } \\
\text { cintura }\end{array}$ & $\geq 150 \mathrm{mg} / \mathrm{dL}$ & $<40 \mathrm{mg} / \mathrm{dL}$ & $\begin{array}{c}>130 \mathrm{mmHg} \\
\text { sistólica o } \\
85 \mathrm{mmHg} \\
\text { diastólica }\end{array}$ & $\begin{array}{c}\text { Glucemia basal } \\
>100 \mathrm{mg} / \mathrm{dL} \mathrm{o} \\
\text { postprandial }> \\
140 \mathrm{mg} / \mathrm{dL}\end{array}$ & $\begin{array}{c}\text { Obesidad abdominal } \\
\text { más otros dos criterios } \\
\text { en adolescentes de } 10 \mathrm{a} \\
16 \text { años }\end{array}$ \\
\hline
\end{tabular}

Tabla I. Criterios de evaluación del SM en niños y adolescentes.

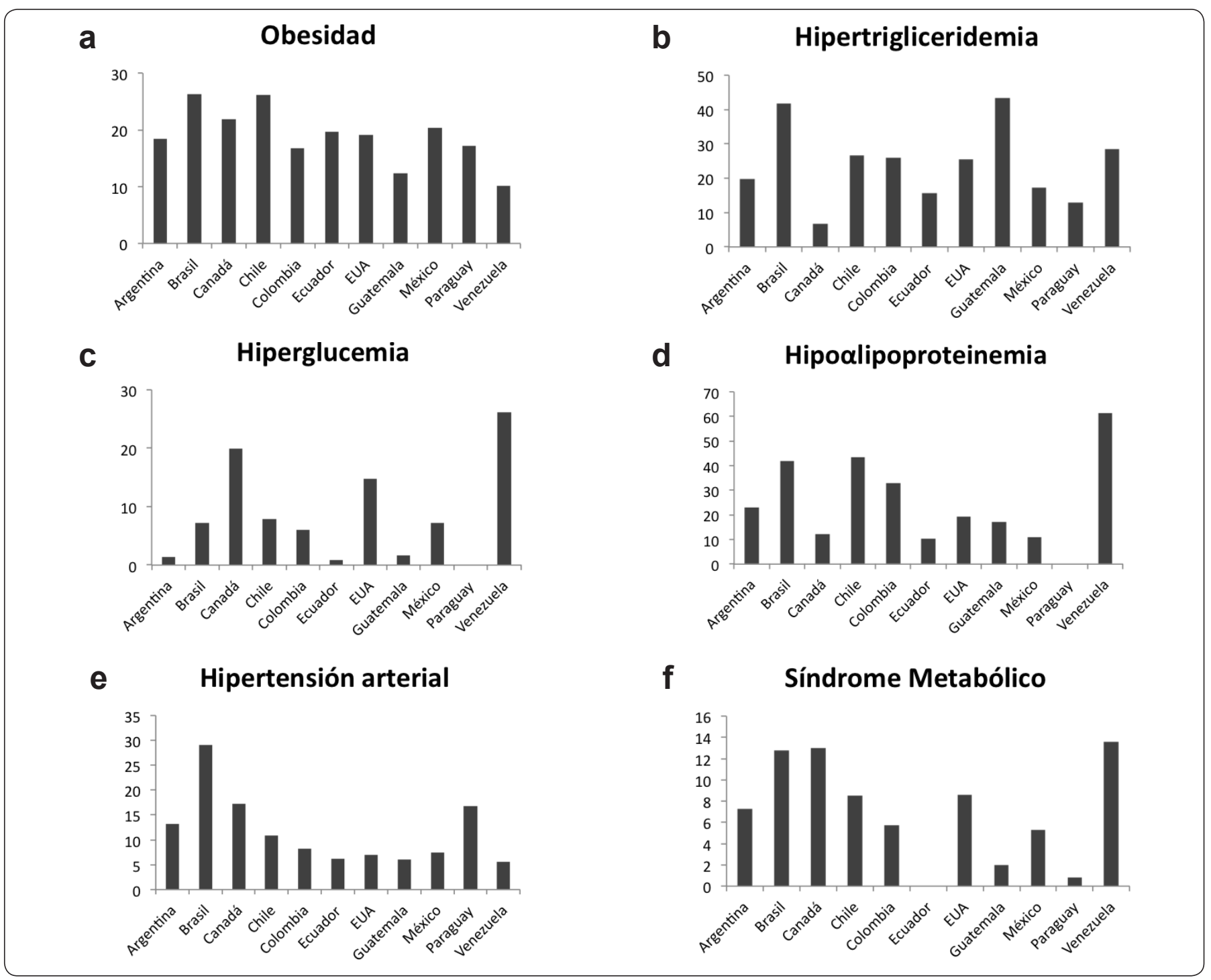

Figura 1. Prevalencias (\%) del SM y sus componentes en niños y adolescentes americanos. Los valores graficados corresponden a los valores reportados en los estudios analizados; excepto para países como Argentina, Brasil, Colombia y México donde se graficó el promedio de los valores reportados en los estudios. 
La comparación de los componentes de SM entre géneros fue realizada en 12 estudios. Los hombres presentaron mayores porcentajes del SM, HDL-C, hiperglucemia e hipertensión arterial (Figuras 2a-f). En 16 estudios se analizaron factores asociados con las variables metabólicas como son obesidad, antecedentes familiares, el lugar de residencia, peso al nacimiento y dieta. Sólo en 3 estudios se analizaron los datos por rango de edad (Tabla II).

\section{Discusión}

Comparando las prevalencias del SM en niños y adolescentes de América, encontramos que existe una importante variabilidad entre los países, siendo las prevalencias menores del 6.0\% para países como Argentina, Colombia, Guatemala,
México y Paraguay. Mientras que las mayores prevalencias fueron para Brasil, Canadá y Venezuela $(>12.0 \%)$. Esta variabilidad es encontrada también en países de otros continentes como es el caso de China con prevalencia del SM baja en niños de primaria con $3.5 \%^{38}$. Otro país es Turquía, donde se ha reportado una prevalencia del SM de $2.3 \%$ en adolescentes de $10-19$ años $^{39}$ o de $2.5-4.4 \%$ en niños de primaria y secundaria dependiendo del método de diagnóstico utilizado ${ }^{40}$. De igual manera, Irán tiene una prevalencia del SM de $5.3 \%$ en niños de 6 a 11 años ${ }^{41}$, de $6.5 \%$ en adolescentes entre 15 a 18 años $^{42}$, y de $4.4 \%$ en adolescentes de $10-18$ años $^{43}$. Mientras que las prevalencias del SM altas han sido reportadas en países como Italia (niños y adolescentes de 6 a 14 años con $13.0 \%)^{44}$.

\section{a}

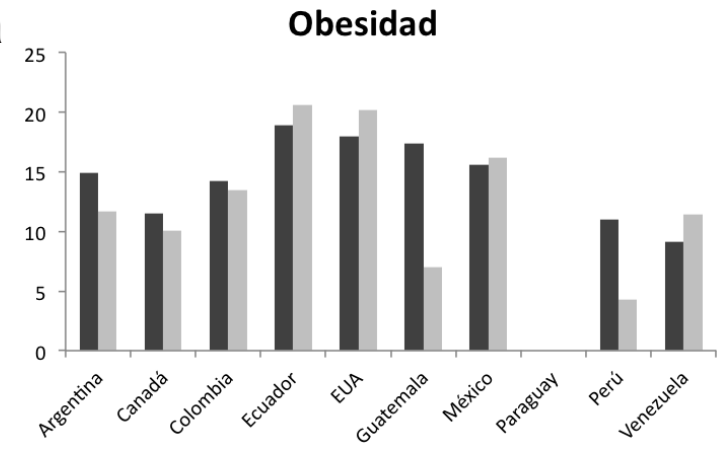

C

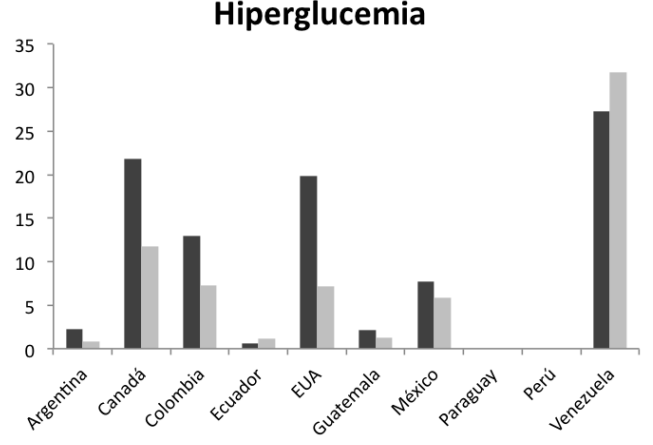

e

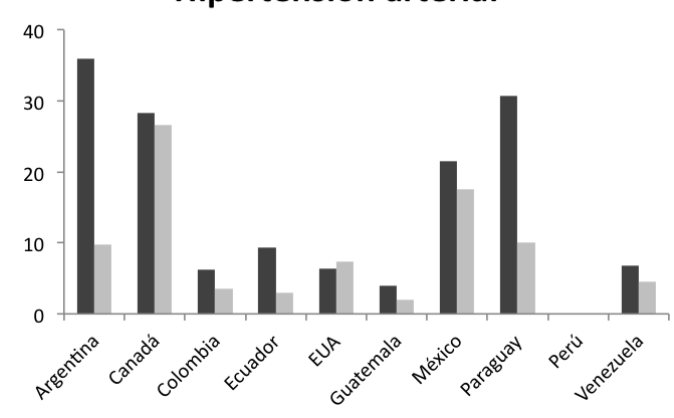

b

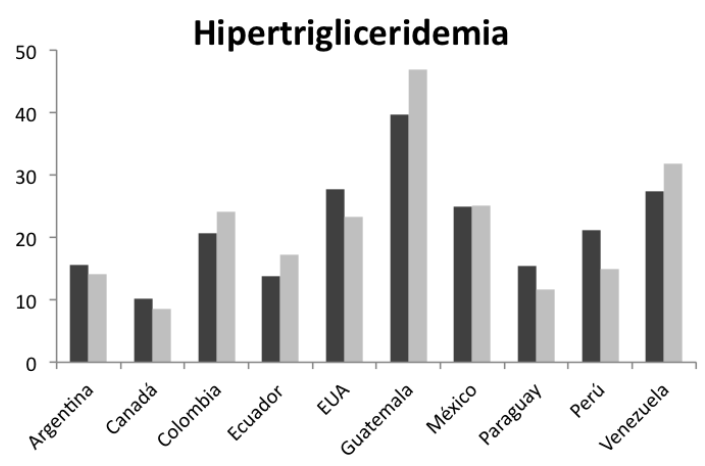

d

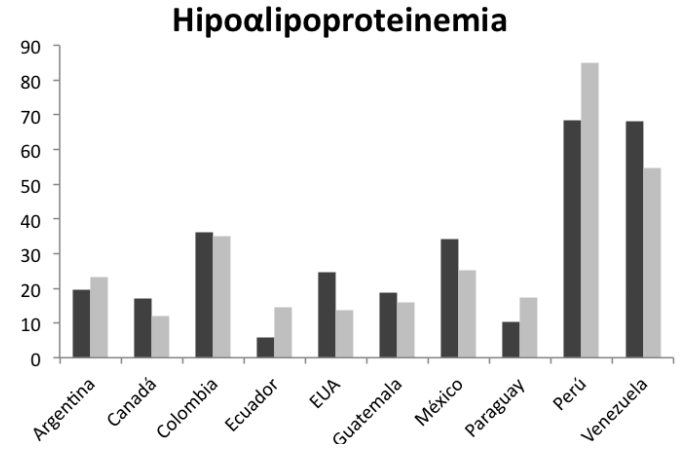

f

Síndrome Metabólico

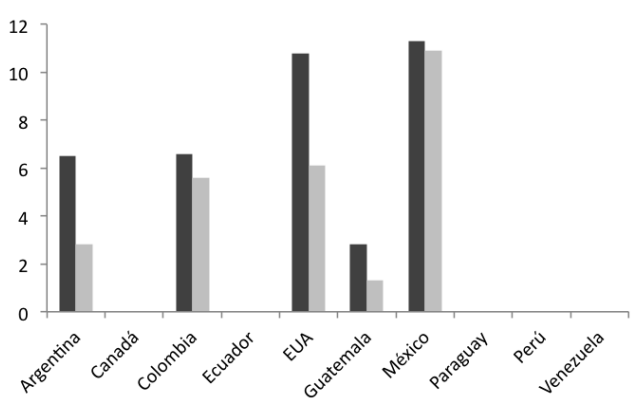

Figura 2. Prevalencias (\%) del SM y sus componentes en niños y adolescentes americanos clasificados por género: hombres (barras negras) y mujeres (barras grises). Los valores graficados corresponden a los valores reportados en los estudios analizados; excepto para países como Argentina, Brasil, Colombia y México donde se graficó el promedio de los valores reportados por lo estudios. 


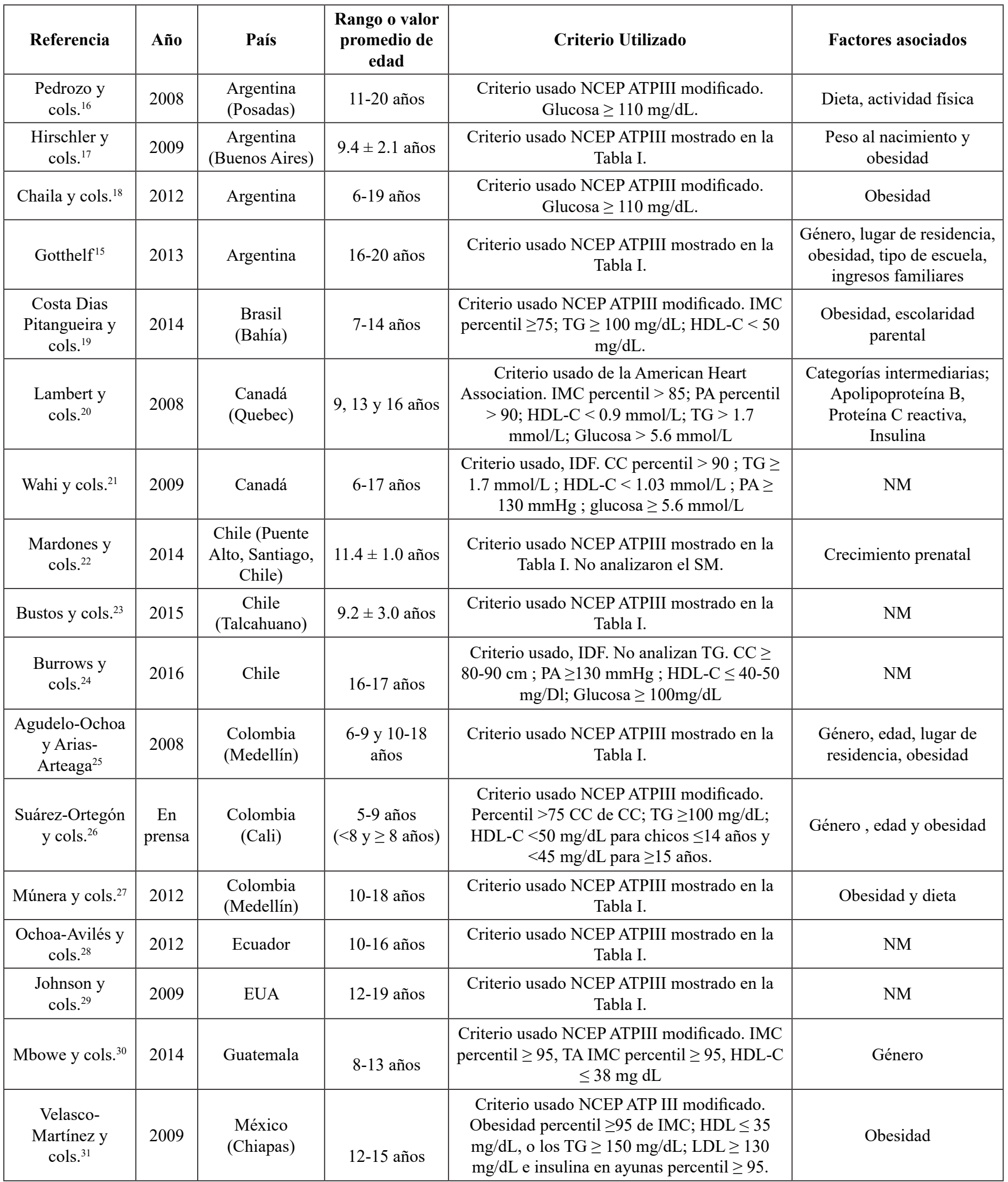

Tabla Il. Criterios usados para el diagnóstico de SM en niños y adolescentes de América y los factores relacionados. Abreviaturas: índice de masa corporal, IMC; triglicéridos, TG; presión arterial, PA; circunferencia de cintura, CC; hipertensión, HTA; no mencionado en el artículo, NM. 


\begin{tabular}{|c|c|c|c|c|c|}
\hline $\begin{array}{l}\text { Cárdenas- } \\
\text { Villarreal y } \\
\text { cols. }^{32} \\
\end{array}$ & 2010 & $\begin{array}{l}\text { México } \\
\text { (Monterrey) }\end{array}$ & 10-19 años & Criterio usado IDF mostrado en la Tabla I. & Obesidad \\
\hline $\begin{array}{c}\text { Cardoso- } \\
\text { Saldaña y cols. }^{33}\end{array}$ & 2010 & México (DF) & 12-16 años & $\begin{array}{l}\text { Criterio usado NCEP ATP III modificado. } \\
\text { Glucosa } \geq 110 \mathrm{mg} / \mathrm{dL} .\end{array}$ & Obesidad \\
\hline Vashi y cols. ${ }^{34}$ & 2016 & $\begin{array}{l}\text { México } \\
\text { (DF) }\end{array}$ & 6-14 años & $\begin{array}{c}\text { Criterio usado NCEP ATPIII modificado. TG } \\
\geq 100 \mathrm{mg} / \mathrm{dL}(0-9 \text { años }) \text { y TG } \geq 130 \mathrm{mg} / \mathrm{dL} \\
(10-19 \text { años }) ; \text { HDL-C }<35 \mathrm{mg} / \mathrm{dL} \text { TA } 140 / 90 \\
\mathrm{mmHg} .\end{array}$ & $\mathrm{NM}$ \\
\hline $\begin{array}{l}\text { Riveros-Sasaki } \\
\text { y cols. }^{35}\end{array}$ & 2012 & Paraguay & 15-19 años & $\begin{array}{l}\text { Criterio usado NCEP ATPIII mostrado en la } \\
\text { Tabla I. }\end{array}$ & $\begin{array}{l}\text { Antecedentes familiares: } \\
\text { HTA y obesidad }\end{array}$ \\
\hline Sáez y Vernui ${ }^{36}$ & 2009 & $\begin{array}{l}\text { Perú } \\
\text { (Lima) }\end{array}$ & 12-17 años & $\begin{array}{c}\text { Criterio usado NCEP ATPIII modificado. TA } \\
\text { percentil } \geq 95, \mathrm{TG} \geq 130 \mathrm{mg} / \mathrm{dL} ; \text { glucosa } \geq 110 \\
\mathrm{mg} / \mathrm{dL} .\end{array}$ & Sedentarismo, dieta \\
\hline
\end{tabular}

Tabla II. Criterios usados para el diagnóstico de SM en niños y adolescentes de América y los factores relacionados. Abreviaturas: índice de masa corporal, IMC; triglicéridos, TG; presión arterial, PA; circunferencia de cintura, CC; hipertensión, HTA; no mencionado en el artículo, NM (continuación).

Las altas prevalencias del SMy sus componentes encontradas en niños y jóvenes del continente americano como ya se mencionó están relacionadas con cambios en la alimentación, estilo de vida, actividad física y nivel socioeconómico. A continuación haremos referencia a la importancia de cada factor.

Influencia del género. En lo que concierne a las diferencias entre hombres y mujeres, encontramos un patrón donde los hombres tuvieron mayores prevalencias del SM y de algunos de sus componentes como la hiperglucemia, la hipertensión arterial y el HDL-C bajo, particularmente en México, Canadá, Colombia y EUA. Otros autores también han reportado que los hombres tienen mayor prevalencia del SM que las mujeres ${ }^{45}$. Además, las adolescentes tienen mayores niveles de insulina que los varones ${ }^{46}$. Ésto podría ser útil para el control de la glucemia, aunque puede favorecer la resistencia a la insulina. Si bien las mujeres jóvenes suelen ser más sedentarias que los hombres, estos últimos fuman e ingieren más alcohol lo que favorece la presencia de alteraciones metabólicas ${ }^{45}$.

Dieta. La ingesta calórica alta y el mayor consumo de carbohidratos en los adolescentes son asociados con el aumento de triglicéridos y HDL-bajo ${ }^{47}$. La ingesta calórica alta, el bajo contenido vitamínico y mineral de los alimentos consumidos favorecen la aparición de alteraciones metabólicas a edad temprana. La deficiencia de vitaminas A y D, zinc (Zn), y magnesio $(\mathrm{Mg})$ han sido un factor de riesgo asociado al SM y alteraciones metabólicas en niños y adolescentes ${ }^{38,48}$. Cambios en la dieta son particularmente importantes para muchos países de América latina, porque favorecen el sobrepeso y la obesidad. Por ejemplo, entre 1964 y 1996, se ha incrementado drásticamente el consumo de grasas. Así, la energía requerida en la dieta proveniente de grasas pasó del 16 a1 28\% en Brasil, del 19 a1 $24 \%$ en México y del 21 a1 24\% en el Caribe. Respecto al colesterol disponible en la comida, éste aumentó casi tres veces en México y dos veces en Brasil ${ }^{49}$. El aumento en el consumo de bebidas azucaradas también es uno de los problemas en la transición alimentaria. En EUA y México, la mayor parte del azúcar de la dieta proviene de las bebidas azucaradas ${ }^{50}$, en EUA, entre el 55.0 al 70.0\% de su consumo ocurre en el hogar y sólo del 7.0 al $15.0 \%$ en la escuela ${ }^{51}$. Respecto a la obesidad abdominal, alrededor del $17 \%$ de los adolescentes del continente americano presentan esta alteración, siendo un problema de salud pública que se ha incrementado de manera alarmante en las últimas décadas. Por ejemplo, en el periodo de 1980 a 2008, la prevalencia de obesidad general en adolescentes de EUA aumentó del 5 al 18\%, existiendo grupos étnicos con cifras significativamente mayores. La obesidad general de los adolescentes varones con ascendencia hispana incrementó del 14.0 al 26.8\% y en las mujeres del 13.4 al 17.4\% $\%^{52}$. En los países en desarrollo, las poblaciones con ingreso elevado tienden a presentar prevalencias de obesidad y sobrepeso más altas ${ }^{53}$. Esto es debido a que han adoptado estilos de vida comparables a las poblaciones de los países desarrollados, consumiendo más calorías en la dieta y realizando menos actividad física. Por su parte, en los países con bajos ingresos, la falta de acceso a las infraestructuras deportivas, educación, alimentación adecuada y recursos que les permitan reducir el riesgo de ganar peso y sus consecuencias metabólicas agrava la situación ${ }^{54}$. Aunado a la dieta, las actitudes y estilos de alimentación son 
importantes. Por ejemplo, los franceses, en comparación con los estadounidenses, consumen frecuentemente productos frescos, planean la comida y su preparación, comen juntos, comen menos y más lentamente ${ }^{55}$, reflejándose en menor peso corporal y dislipidemias.

Actividad física. La actividad física moderada y vigorosa evita el desarrollo del SM, incluso más que la reducción calórica ${ }^{56}$. Considerando este factor, los niños y adolescentes americanos tienen poca actividad física como lo muestran algunos estudios. En Canadá, menos del $10.0 \%$ de los jóvenes y niños siguen la recomendación de realizar al menos 60 minutos de actividad física al día ${ }^{57}$. En los EUA, en el año 2006, se reportó que el $61.5 \%$ de los adolescentes entre 9 a 13 años de edad participaban en actividades físicas fuera de la escuela ${ }^{58}$. En el año 2008, en las escuelas públicas mexicanas, se reportó que sólo un tercio de los jóvenes (28.0\%) cumplen con los requisitos de actividad física ${ }^{59}$. Por su parte, en países europeos, los varones de 12.5 a 17.5 años de edad hacen en promedio 70 minutos de actividad moderada a vigorosa a la semana $v s$. las mujeres que realizan 50 minutos. Por ello, más del 55.0\% de los varones cumplen con las recomendaciones de 60 minutos de actividad física moderada a vigorosa al día vs. el 25.0\% de las mujeres. Así, se ha mostrado que varones con índice de masa corporal (IMC) alto realizan menos actividad física ${ }^{60}$. La cultura es un factor decisivo en la realización de actividad física. Por ejemplo, los franceses dedican más tiempo a caminar ( $1.6 \%$ de su tiempo) que los estadounidenses ( $0.6 \%$ de su tiempo). Los estadounidenses tienden a preferir los deportes de intensidad elevada, lo que trae consigo mayor probabilidad de lesiones y periodos de sedentarismo prolongados ${ }^{55}$.

Antecedentes familiares, peso al nacimiento y hábitos familiares. La hiperglucemia y la hipertensión tienen una clara relación con la herencia genética de los individuos ${ }^{61-62}$. La presión arterial y la hiperglicemia, así como las dislipidemias también dependen del peso al nacimiento, que refleja como fue el desarrollo intrauterino de los individuos. Así, un peso al nacimiento alto o bajo se consideran factores de riesgo para el desarrollo del SM en la niñez y la adolescencia. El peso al nacimiento puede correlacionarse con la presión diastólica, glucosa sérica, lipoproteína de baja densidad (LDL-C), obesidad y HDL-C ${ }^{63}$. La obesidad y las dislipidemias también dependen del entorno familiar en el que vive un individuo. Esto es importante particularmente en los niños y jóvenes, los cuales son cautivos de los hábitos de alimentación y actividad física que realizan sus padres ${ }^{1}$.

Esta revisión, tuvo algunas limitaciones como lo es la carencia de datos específicos por grupos de edad, las diferencias en los criterios metodológicos para medir el SM y sus componentes, y la disparidad en la representatividad de las muestras poblacionales estudiadas, lo que dificultó poder profundizar en el análisis comparativo entre las prevalencias de las enfermedades metabólicas en jóvenes de las distintas regiones y países de América. Desafortunadamente, los estudios incluidos en esta revisión no coincidieron con las edades analizadas ni con los criterios para considerar infancia y adolescencia. Muchos de ellos incluso analizaron niños y adolescentes como un solo grupo, lo que dificulta dilucidar el impacto de las hormonas sexuales en la aparición de los componentes del SM. En cierta medida, parte de esta confusión se debe a los criterios utilizados en el diagnóstico del SM, los cuales están basados en lo que ocurre en adultos y no en estudios pediátricos ${ }^{2}$, sin embargo, a pesar de las limitaciones, el análisis realizado nos permitió evaluar la prevalencia del SMy sus componentes en los países del continente americano y compararlos con países de otros continentes. Esto podría ayudar a resaltar la importancia de implementar políticas públicas diferenciadas a la cultura y origen que permitan promover la salud y el bienestar de las poblaciones de jóvenes americanos. Para ello, se requiere facilitar el acceso a alimentos saludables y a un costo accesible, así como a la infraestructura deportiva que incentive la adopción de un estilo de vida saludable. Al mismo tiempo, esta revisión apoya la necesidad de realizar estudios epidemiológicos en niños y adolescentes específicos para cada edad, y criterios de diagnóstico del SM y sus componentes más claros.

\section{CONCLUSIONES}

El presente artículo muestra que la prevalencia del SM y sus componentes en diversos países del continente americano es variable, dependiendo de cada país. Hay países con prevalencias pequeñas, pero hay otros con prevalencias altas. Esta variabilidad también es observada en países de otros continentes, por lo que posiblemente se deba a la diferencia en los hábitos alimenticios y cuidados de la salud que cada país tiene, pero también podría depender de la diversidad de criterios utilizados en el diagnóstico y puntos de corte para los componentes del SM. Por lo que la presente revisión permite observar la falta de consenso entre los investigadores y las instituciones responsables de establecer los criterios de detección.

\section{Agradecimientos}

Este estudio forma parte del Programa de Salud promovido por la Estación Científica La Maliche, Universidad Nacional Autónoma de México-Universidad Autónoma de Tlaxcala. Los autores agradecen la excelente asistencia de Laura García.

\section{Referencias}

1. Ewald, D. R. \& Haldeman, L. A. Risk factors in adolescent hypertension. Glob. Pediatr. Health 3, 2333794X15625159 (2016). DOI: 10.1177/2333794X15625159.

2. Reinehr, T. Metabolic syndrome in children and adolescents: a critical approach considering the interaction between pubertal stage and insulin resistance. Curr. Diab. Rep. 16(1), 8-17 (2016). DOI: $10.1007 / \mathrm{s} 11892-015-0695-1$.

3. Moreno Giraldo, L. J., Velasco-Benítez, C. A. \& Matallana Rhoades, A. M. Síndrome Metabólico en niños y adolescentes. Revista Gastrohnup 16(2), 121-131 (2014).

4. Bouzas, I. C., Cader, S. A., Leão, L., Kuschnir, M. C. \& Braga, C. 
Menstrual cycle alterations during adolescence: early expression of metabolic syndrome and polycystic ovary syndrome. J. Pediatr. Adolesc. Gynecol. 27(6), 335-341 (2014). DOI: 10.1016/j. jpag.2014.01.002.

5. Kim, J. Y., Tfayli, H., Michaliszyn, S. F., Lee, S. \& Arslanian, S. Distinguishing characteristics of metabolically healthy versus metabolically unhealthy obese adolescent girls with polycystic ovary syndrome. Fertil. Steril 105(6), 1603-1611 (2016). DOI: 10.1016/j.fertnstert.2016.02.004.

6. McPherson, N.O. \& Lane, M. Male obesity and subfertility, is it really about increased adiposity? Asian J. Androl. 17(3), 450-458 (2015). DOI: 10.4103/1008-682X.148076.

7. Lee, H. S. Impact of maternal diet on the epigenome during in utero life and the developmental programming of diseases in childhood and adulthood. Nutrients 7(11), 9492-9507 (2015). DOI: $10.3390 /$ nu7115467.

8. Keane, E., Layte, R., Harrington, J., Kearney, P. M. \& Perry, I. J. Measured parental weight status and familial socio-economic status correlates with childhood overweight and obesity at age 9. PLoS One 7(8), e43503 (2012). DOI: 10.1371/journal.pone.0043503.

9. Centers for Disease Control and Prevention, National Center for Chronic Disease Prevention and Health Promotion, Division of Adolescent and School Health; Health Resources and Services Administration, Maternal and Child Health Bureau, Office of Adolescent Health; National Adolescent Health Information Center, University of California, San Francisco. Improving the health of adolescents \& young adults: A guide for states and communities. Atlanta, Ga. 2004.

10. Lawrence, R. ,S., Gootman, J. A. \& Sim, L. J. Adolescent Health Services: Missing Opportunities (National Academies Press, Washington, DC, 2009). National Research Council (U.S.).

11. PAHO.Adolescents \& non-communicable diseases. (Panamerican Health Organization, Washington, DC, 2011). www.paho.org/ncds

12. World Health Organization. The world health report 2002: Reducing risks, promoting healthy life (World Health Organization, Geneva, 2002)

13. Cook, S., Weitzman, M., Auinger, P., Nguyen, M. \& Dietz, W. H. Prevalence of a metabolic syndrome phenotype in adolescents: findings from the third National Health and Nutrition Examination Survey, 1988-1994. Arch. Pediatr. Adolesc. Med. 157(8), 821827 (2003).

14. Zimmet, P. Alberti, G., Kaufman, F., Tajima, N., Silink, M., Arslanian, S., Wong, G., Bennett, P., Shaw, J. \& Caprio, S. The metabolic syndrome in children and adolescents. Lancet 369(9579), 2059-2061 (2007).

15. Gotthelf, S. Prevalence of metabolic syndrome as defined by the International Diabetes Federation (IDF) in adolescents from Salta, Argentina. Rev. Fed. Arg. Cardiol. 42(2), 119-126 (2013).

16. Pedrozo, W., Rascón, M. C., Bonneau, G., Ibáñez de Pianesi, M., Castro Olivera, C., Jiménez de Aragón, S., Ceballos, B. \& Gauvry, G. Síndrome metabólico y factores de riesgo asociados con el estilo de vida de adolescentes de una ciudad de Argentina, 2005. Rev. Panam. Salud Pública 24(3), 149-160 (2008).

17. Hirschler, V., Silva, S., Gilligan, T., Bugna, J. \& Dalamón, R. Relationship between birth weight and obesity and metabolic syndrome among school children. Actualización en Nutrición 10(1), 37-44 (2009).

18. Chaila, M. Z., Fabio, S., Quiroga, E., Sánchez de Boeck, N.,
Namur, M., D’urso, M. \& Bazán, M. C. Prevalencia de síndrome metabólico en niños y adolescentes según diferentes criterios diagnósticos y su correlación con niveles de leptina y adiponectina. Rev. Argent. Endocrinol. Metab. 49(3), 103-114 (2012).

19. Costa Dias Pitangueira, J., Silva, L. R., Portela de Santana, M. L., da Conceição Monteiro da Silva, M., Ribas de Farias Costa, P., D'Almeida, V. \& de Oliveira Assis, A. M. Síndrome metabólico y factores asociados en niños y adolescentes de un municipio brasileño. Nutr. Hosp. 29(4), 865-872 (2014).

20. Lambert, M., Delvin, E. E., Levy, E., O’Loughlin, J., Paradis, G., Barnett, T. \& McGrath, J. J. Prevalence of cardiometabolic risk factors by weight status in a population-based sample of Quebec children and adolescents. Can. J. Cardiol. 24(7), 575-583 (2008).

21. Wahi, G.,Zorzi,A., Macnab, A.\& Panagiotopoulos, C. Prevalence of type 2 diabetes, obesity and the metabolic syndrome among Canadian First Nations children in a remote Pacific coast community. Paediatr. Child. Health 14(2), 79-83 (2009).

22. Mardones, F., Arnaiz, P., Pacheco, P., Domínguez, A., Villarroel, L., Eriksson, J. G., Barja, S., Farías, M. \& Castillo, O. Associations of prenatal growth with metabolic syndrome, insulin resistance, and nutritional status in Chilean children. Biomed. Res. Int., 2014, 472017 (2014). DOI: 10.1155/2014/472017.

23. Bustos, P., Orias, J., Sáez, K., Maldonado, M., Cuadra, L. \& Asenjo, S. Impacto del Programa de manejo de la obesidad Bright Bodies aplicado a niños y adolescentes chilenos. Rev. Med. Chile 143(9), 1136-1143 (2015).

24. Burrows, R., Correa-Burrows, P., Reyes, M., Blanco, E., Albala, C. \& Gahagan, S. High cardiometabolic risk in healthy Chilean adolescents: associations with anthropometric, biological and lifestyle factors. Public. Health. Nutr. 19(03), 486-493 (2016). DOI: $10.1017 / \mathrm{S} 1368980015001585$.

25. Agudelo Ochoa, G. M. \& Arias Arteaga, R. Prevalencia del síndrome metabólico en niños y adolescentes escolarizados delárea urbana de la ciudad de Medellín. Iatreia 21(3), 260-270 (2008).

26. Suárez-Ortegón, M. F. \& Aguilar-de Plata, C. Prevalence of metabolic syndrome in children aged 5-9 years from southwest colombia: a cross-sectional study. World. J. Pediatr. En prensa. DOI:10.1007/s12519-016-0008-Z.

27. Múnera, N. E., Uscátegui, R., Parra, B., Manjarrés, L., Patiño, F., Velásquez, C., Estrada, A., Bedoya, G., Parra, V., Muñoz, A., Orozco, A. \& Agudelo, G. Factores de riesgo ambientales y componentes del síndrome metabólico en adolescentes con exceso de peso. Biomédica 32(1), 77-91 (2012).

28. Ochoa-Avilés, A., Andrade, S., Huynh, T., Verstraeten, R., Lachat, C., Rojas, R., Donoso, S., Manuel-Y-Keenoy, B. \& Kolsteren, P. Prevalence and socioeconomic differences of risk factors of cardiovascular disease in Ecuadorian adolescents. Pediatr. Obes. 7(4), 274-283 (2012). DOI: 10.1111/j.2047-6310.2012.00061.x

29. Johnson, W. D. Kroon, J. J., Greenway, F. L., Bouchard, C., Ryan, D. \& Katzmarzyk, P. T. Prevalence of risk factors for metabolic syndrome in adolescents. National Health and Nutrition Examination Survey (NHANES), 2001-2006. Arch. Pediatr. Adolesc. Med. 163(4), 371 (2009). DOI: 10.1001/ archpediatrics.2009.3.

30. Mbowe, O., Díaz, A., Wallace, J., Mazariegos, M. \& Jolly, P. Prevalence of metabolic syndrome and associated cardiovascular risk factors in Guatemalan school children. Matern. Child. Health J. 18(7), 1619-1627 (2014). DOI: 10.1007/s10995-013-1402-y. 
31. Velasco-Martínez, R. M., Jiménez-Cruz,A., Higuera Domínguez, F., Domínguez de la Piedra, E. \& Bacardí-Gascón, M. Obesidad y resistencia a la insulina en adolescentes de Chiapas. Nutr. Hosp. 24(2), 187-192 (2009).

32. Cárdenas-Villarreal, V. M., López-Alvarenga, J.C., Bastarrachea, R. A., Rizo-Baeza, M. M. \& Cortés-Castell, E. Prevalencia del síndrome metabólico y sus componentes en adolescentes de la Ciudad de Monterrey, Nuevo León. Arch. Cardiol. Mex. 80(1), 19-26 (2010).

33. Cardoso-Saldaña, G. C., Yamamoto-Kimura, L., Medina-Urrutia, A., Posadas-Sánchez, R., Caracas-Portilla, N.A. \& PosadasRomero, C. Exceso de peso y síndrome metabólico en adolescentes de la Ciudad de México. Arch. Cardiol. Mex. 80(1), 12-18 (2010).

34. Vashi, N. , Stryjecki, C., Peralta-Romero, J., Suárez, F., GómezZamudio, J., Burguete-García,A. I., Cruz, M. \& Meyre, D. Genetic markers of inflammation may not contribute to metabolic traits in Mexican children. PeerJ. 4, e2090 (2016). DOI: 10.7717/ peerj.2090.

35. Riveros Sasaki, K., Alderete Peralta, V. \& Sánchez Bernal, S. Frecuencia de elementos del Síndrome Metabólico en adolescentes de un colegio público. Pediatría (Asunción). 39(1), 13-19(2012).

36. Saez, Y. \& Bernui, I. Prevalencia de factores de riesgo cardiovascular en adolescentes de instituciones educativas. An. Fac. Med. 70(4), 259-265 (2009).

37. Lozada, M., Machado, S., Manrique, M., Martínez, D., Suárez, O. \& Guevara, H. Factores de riesgo asociados al síndrome metabólico en adolescentes. Gac. Med. Caracas 116(4), 323-329 (2008).

38. Wei, X., Peng, R., Cao, J., Kang, Y., Qu, P., Liu, Y., Xiao, X. \& $\mathrm{Li}, \mathrm{T}$. Serum vitamin A status is associated with obesity and the metabolic syndrome among school-age children in Chongqing, China. Asia Pac. J. Clin. Nutr. 25(3), 563-570 (2016). DOI: 10.6133/apjen.092015.03.

39. Bereket, A. \& Atay, Z. Current status of childhood obesity and its associated morbidities in Turkey.J. Clin. Res. Pediatr. Endocrinol. 4(1), 1-7 (2012). DOI: 10.4274/jcrpe.506.

40. Agirbasli, M., Agaoglu, N. B., Orak, N., Caglioz, H., Ocek, T., Poci, N., Salaj, A. \& Maya, S. Sex hormones and metabolic syndrome in children and adolescents. Metabolism. 58(9), 12561262 (2009). DOI: 10.1016/j.metabol.2009.03.024.

41. Zardast, M., Namakin, K., Chahkandi, T., Taheri, F., Kazemi, T. \& Bijari, B. Prevalence of metabolic syndrome in elementary school children in east of Iran. J. Cardiovasc. Thorac. Res. 7(4), 158-163 (2015). DOI: 10.15171/jevtr.2015.34.

42. Mirhosseini, N. Z., Yusoff, N. A. M., Shahar, S., Parizadeh, S. M. R., Mobarhen, M. G. \& Shakery, M. T. Prevalence of the metabolic syndrome and its influencing factors among adolescent girls in Mashhad, Iran. Asia Pac. J. Clin. Nutr. 18(1), 131-136 (2009).

43. Shafiee, G., Kelishadi, R., Heshmat, R., Qorbani, M., Motlagh, M. E., Aminaee, T., Ardalan, G., Taslimi, M., Poursafa, P. \& Larijani, B. First report on the validity of a continuous metabolic syndrome score as an indicator for metabolic syndrome in a national sample of paediatric population - the CASPIAN-III study. Endokrynol. Pol. 64(4), 278-284 (2013).

44. Martino, F., Pannarale, G., Puddu, P. E., Colantoni, C., Zanoni, C., Martino, E., Torromeo, C., Paravati, V., Perla, F. M. \& Barillà, F. Is it possible a new definition of metabolic syndrome in childhood? Eur. Rev. Med. Pharmacol. Sci. 19(22), 4324-4331 (2015).

45. Barbosa, J. B., Santos, A. M. D., Barbosa, M. M., Carvalho, C.
A. D., Fonseca, P. C. D. A., Fonseca, J. M., Barbosa, M. D. C. L., Bogea, E. G. \& Silva, A. A. M. D. Metabolic syndrome, insulin resistance and other cardiovascular risk factors in university students. Cien. Saude Colet. 21(4), 1123-1136 (2016). DOI: 10.1590/1413-81232015214.10472015.

46. DeBoer, M. D., Dong, L. \& Gurka, M. J. Racial/ethnic and sex differences in the ability of metabolic syndrome criteria to predict elevations in fasting insulin levels in adolescents. J. Pediatr. 159(6), 975-981.e3 (2011). DOI: 10.1016/j.jpeds.2011.05.023.

47. Lee, A. M., Gurka, M. J. \& DeBoer, M. D. Trends in metabolic syndrome severity and lifestyle factors among adolescents. Pediatrics 137(3), e20153177 (2016). DOI: 10.1542/peds.20153177.

48. Khosravi-Boroujeni, H., Ahmed, F. \& Sarrafzadegan, N. Is the association between vitamin $\mathrm{D}$ and metabolic syndrome independent of other micronutrients. Int. J. Vitam. Nutr. Res. 20, 1-16 (2016).

49. Bermúdez, O.I. \& Tucker, K.L. Trends in dietary patterns of Latin American populations. Cad. Saude Publica 19(1 supl), 87-99 (2003).

50. Proper, K. I. \& van Mechelen, W. Effectiveness and economic impact of worksite interventions to promote physical activity and healthy diet. Technical paper prepared for the WHO/World Economic Forum joint event on preventing non communicable diseases in the workplace. (World Health Organization, Geneva, 2008).

51. Wang, Y.C., Bleich, S.N. \& Gortmaker, S.L. Increasing caloric contribution from sugar-sweetened beverages and $100 \%$ fruit juices among US children and adolescents, 1988-2004. Pediatrics 121(6), e1604-e1614 (2004). DOI: 10.1542/peds.2007-2834.

52. Ogden, C. \& Carroll, M. Prevalence of obesity Among Children and Adolescents 2007-2008. Division of Health and Nutrition Examination Surveys Centers for Disease Control and Prevention 2010. National Center for Health Statistics. http://www.cdc.gov/ nchs/data/hestat/obesity_child_07_08/obesity_child_07_08.htm

53. Xie, B., Gilliland, F. D., Li, Y. F. \& Rockett, H. R. Effects of ethnicity, family income, and education on dietary intake among adolescents. Prev. Med. 36(1), 3040 (2003).

54. Popkin, B. M. The world is fat. Sci. Am. 297(3), 88-95 (2007).

55. Powell, L. H., Kazlauskaite, R., Shima, C. \& Appelhans, B. M. Lifestyle in France and the United States: an American perspective. J. Am. Diet Assoc. 110(6), 845-847 (2010). DOI: 10.1016/j. jada.2010.03.029.

56. Frugé,A.D., Byrd, S. H., Fountain, B. J., Cossman, J. S., Schilling, M. W. \& Gerard, P. Increased physical activity may be more protective for metabolic syndrome than reduced caloric intake. An analysis of estimated energy balance in U.S. adults: 2007-2010 NHANES. Nutr. Metab. Cardiovasc. Dis. 25(6), 535-540 (2015). DOI: 10.1016/j.numecd.2015.03.006.

57. Garriguet, D. \& Colley, R. C. Daily patterns of physical activity among Canadians. Health Rep. 23(2), 27-32 (2012).

58. Huhman, M. Lowry, R., Lee, S. M., Fulton, J. E., Carlson, S. A. \& Patnode, C. D. Physical activity and screen time: Trends in U.S. children aged 9 to 13 years, 2002-2006.JPAH9(4), 508-515(2012).

59. Shamah Levy, T.\& Palma Coca, O. Encuesta de salud en estudiantes de escuelas públicas en México-Resumen Ejecutivo. (Instituto Nacional de Salud Pública, Cuernavaca, 2008).

60. Ruiz, J. R., Ortega, F. B., Martínez-Gómez, D., Labayen, I., 
Moreno, L.A., De Bourdeaudhuij, I., Manios, Y., González-Gross, M., Mauro, B., Molnar, D., Widhalm, K., Marcos, A., Beghin, L., Castillo, M. J., Sjöström, M. \& on behalf of the HELENA Study Group. Objectively measured physical activity and sedentary time in european adolescents the HELENA study. Am. J. Epidemiol. 174(2), 173-184 (2011). DOI: 10.1093/aje/kwr068.

61. Katulanda, P., Ranasinghe, P., Jayawardena, R., Sheriff, R. \& Matthews, D. R. The influence of family history of diabetes on disease prevalence and associated metabolic risk factors among Sri Lankan adults. Diabet. Med. 32(3), 314-323 (2015). DOI: 10.1111/dme.12591.
62. Ranasinghe, P., Cooray. D. N., Jayawardena, R. \& Katulanda, P. The influence of family history of hypertension on disease prevalence and associated metabolic risk factors among Sri Lankan adults. BMC Public Health 20(15), 576-585 (2015). DOI: 10.1186/s12889-015-1927-7.

63. Ramadhani, M. K., Grobbee, D. E., Bots, M. L., Cabezas, M. C., Vos, L. E., Oren, A. \& Uiterwaal, C. S. Lower birth weight predicts metabolic syndrome in young adults: the Atherosclerosis Risk in Young Adults (ARYA)-study. Atherosclerosis 184(1), 21-27 (2006). 